\section{Epidemiological trends of pre-malignant gastric lesions: a long-term nationwide study in the Netherlands}

\author{
A C de Vries, G A Meijer, C W N Looman, M K Casparie, \\ B E Hansen, N C T van Grieken, E J Kuipers
}

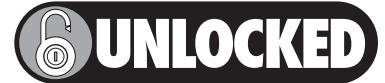

This paper is freely available online under the BMJ Journals unlocked scheme, see http://gut.bmj.com/info/unlocked.dtl
See end of article for authors' affiliations

Correspondence to: A C de Vries, Department of Gastroenterology and Hepatology, Erasmus MC, Room L-462, PO Box 2040, 3000 CA Rotterdam, The Netherlands; A.C.deVries@ erasmusmc.nl

Revised 13 June 2007 Accepted 30 June 2007

Published Online First

14 August 2007
Background: The pre-malignant gastric lesions atrophic gastritis (AG), intestinal metaplasia (IM) and dysplasia (DYS) have long been identified as principal risk factors for gastric cancer.

Objective: To evaluate epidemiological time trends of pre-malignant gastric lesions in the Netherlands.

Methods: Patients with a first diagnosis of AG, IM or DYS between 1991 and 2005 were identified in the Dutch nationwide histopathology registry. The number of new diagnoses per year were evaluated relative to the total number of patients with a first gastric biopsy. Time trends were evaluated with age-period-cohort models using logistic regression analysis.

Results: In total, 23278 patients were newly diagnosed with AG, 65937 patients with IM, and 8517 patients with DYS. The incidence of AG declined similarly in men and women with $8.2 \%$ per year $[95 \% \mathrm{Cl}$ $7.9 \%$ to $8.6 \%$, and DYS with $8.1 \%$ per year [95\% Cl 7.5\% to $8.6 \%$ ]. The proportional number of new $\mathrm{IM}$ cases declined with $2.9 \%$ per year $[95 \% \mathrm{Cl} 2.7 \%$ to $3.1 \%]$ in men and $2.4 \%[95 \% \mathrm{Cl} 2.2 \%$ to $2.6 \%]$ in women. With age-period-cohort models a cohort phenomenon was demonstrated for all categories of pre-malignant gastric lesions in men and in women with IM and DYS. Period phenomena with a larger decline in number of diagnoses after 1996 were also demonstrated for AG and IM.

Conclusions: The incidence of pre-malignant gastric lesions is declining. Period and cohort phenomena were demonstrated for diagnoses of $A G$ and IM. These findings imply that a further decrease of at least $24 \%$ in the incidence of gastric cancer in the coming decade may be anticipated in Western countries without specific intervention.
G astric cancer represents the fourth most common cancer and second leading cause of cancer-related death worldwide. The estimated current incidence of gastric cancer is approximately 16.2/100 000 persons/year (world standardised rate, WSR), with highest incidences in Eastern Asia, Eastern Europe and South America. ${ }^{1}$ For example, in Japan, the incidence of gastric cancer is approximately 44.1 cases/100 000 persons/year (WSR). In comparison, in the Netherlands, the incidence of gastric cancer is relatively low with approximately 6.9 cases/100 000 persons/year (WSR). ${ }^{2}$ Although the incidence of gastric cancer has declined over the past decades, especially in Western countries, mortality remains high. As symptoms are frequently absent or only vague until the disease reaches an advanced stage, curative therapeutic options are usually limited at the time of diagnosis. ${ }^{3}$

The vast majority of gastric malignancies are adenocarcinomas, which can be divided into carcinomas of the intestinal and diffuse (undifferentiated) type. Intestinal type gastric carcinomas account for approximately $60 \%$ of cancers and are generally preceded by a sequence of precursor lesions. This multi-step model of gastric carcinogenesis is strongly associated with Helicobacter pylori colonisation; especially corpus-predominant $H$ pylori-induced gastritis has been identified as an important risk factor for gastric cancer development. ${ }^{4}$ Chronic inflammation of the gastric mucosa can progress through the pre-malignant stages of atrophic gastritis, intestinal metaplasia and dysplasia to eventually gastric adenocarcinoma over a time frame of several years to decades. ${ }^{5-8}$ The recognition of this long-term cascade may provide a basis for early detection, surveillance and treatment of advanced precursors and early gastric carcinomas and thereby improve survival.
The prevalence of $H$ pylori has declined significantly in Western countries during the past decades. ${ }^{9}$ However, since symptoms are most frequently absent in patients with pre-malignant gastric lesions, epidemiology of these lesions is largely unknown, especially in regions with a relatively low incidence of gastric cancer..$^{10}$ Epidemiological data of pre-malignant gastric lesions are, however, relevant as an accurate predictor of gastric cancer incidence in the coming decade, and as determinant in the evaluation of screening and surveillance practices.

Therefore, the aim of the present study was to evaluate the incidence and time trends of atrophic gastritis, intestinal metaplasia and dysplasia in the Netherlands. As reliable incidence data are lacking, the prevalence of pre-malignant conditions as recorded in pathology reports was analysed alternatively.

\section{METHODS}

\section{Histopathology database}

In the Netherlands all histopathology and cytopathology reports are collected in a national archive (PALGA database), which has nationwide coverage since 1991. ${ }^{11}$ Patients in this database are identified by date of birth, gender and the first four characters of their family name. Every record in the database contains a summary of a pathology report and diagnostic codes similar to the Systematized Nomenclature of Medicine (SNOMED) classification of the College of American Pathologists. ${ }^{12}$ The diagnostic code contains a term indicating the anatomical location, type of sample, and a morphological

Abbreviations: $A G$, atrophic gastritis; DYS, dysplasia; $I M$, intestinal metaplasia; OR, odds ratios; PAC, percentual annual changes; WSR, world standardised rate 
term describing the finding, for example, "stomach*biopsy*intestinal metaplasia". Details about the number and intragastric location of biopsies and presence of $H$ pylori are not uniformly registered. After a report has been coded, it is submitted online to the central database. At present, the PALGA database contains 38 million records from approximately 10 million individuals. The present study was based on data recorded in the PALGA database between 1991 and 2005. The following items were made available for each report: gender, date of birth, date of pathology review, summary text and diagnostic code.

\section{Incidence analysis}

All patients with a histopathologically confirmed diagnosis of a pre-malignant gastric lesion were identified in the database. Pre-malignant gastric lesions were defined as atrophic gastritis, intestinal metaplasia or dysplasia. The SNOMED codes that were used to identify the lesions are described in the appendix. For each patient only the most severe pre-malignant lesion at baseline, that is, the first observation of a pre-malignant lesion, was evaluated. This means that patients with atrophic gastritis without a diagnosis of concomitant intestinal metaplasia were classified as having atrophic gastritis, patients with atrophic gastritis and intestinal metaplasia as intestinal metaplasia, and patients with gastric dysplasia as dysplasia.

Patients who had undergone gastric or oesophageal surgery, as far as could be determined from the database, or had been diagnosed with an oesophageal or gastric malignancy prior to or simultaneously with the first diagnosis of a pre-malignant gastric lesion were excluded from analysis.

In order to correct for possible changes in the frequency of upper gastrointestinal (GI) endoscopies with gastric biopsy sampling, rather than prevalence changes of pre-malignant gastric lesions, we also studied the trend in total number of patients with a first time biopsy of the stomach. The ratio of the number of new patients with a positive biopsy for premalignant gastric lesions to the number of new patients with a first time gastric biopsy was determined.

To evaluate the incidence of pre-malignant gastric lesions in different age classes, prevalence numbers in different periods were calculated within five-year age groups.

\section{Statistical analysis}

Age-standardised prevalence rates (WSR) of histopathologically confirmed pre-malignant lesions were calculated for the years 1991-2005. ${ }^{13}$ Prevalence trends were evaluated with age-periodcohort models using logistic regression analysis. This analysis produces odds ratios (ORs) that quantify changes in time. Since these ORs (per year) are always close to 1.00 we present them as percentual annual changes (PAC). Calculations were corrected for age. In the age-period-cohort models, estimated drift parameters reproduce these PACs, which can be interpreted as linear period or cohort changes. For calculating non-linear period and cohort effects, linear splines were used. This means that to assess the presence of a period effect in the incidence of pre-malignant gastric lesions, the linear relationship between year and prevalence was replaced by a line consisting of three joined linear pieces, with knots at 1995 and 2000. For the estimation of cohort models a mean year of birth was calculated for each five-year age group and changes in prevalence were allowed to change in 1920, 1935, 1950 and 1965. Likelihood ratio tests (comparison of scaled deviances) showed whether significant non-linear period or cohort effects were present. ${ }^{14}{ }^{15}$

\section{RESULTS}

Between 1991 and 2005, 97732 patients were newly diagnosed with a pre-malignant gastric lesion, with a l:1 male:female ratio (table 1 ).
The study population consisted of 23278 patients with a first diagnosis of atrophic gastritis as the most severe pre-malignant lesion at initial diagnosis, 65937 patients with intestinal metaplasia and 8517 patients with gastric dysplasia. Significantly more women were present in the atrophic gastritis group (male:female 1.0:1.2) than in the group of patients with intestinal metaplasia (1.0:0.9) or dysplasia (1.0:0.8) $(\mathrm{p}<0.001)$. The median age at diagnosis was 65.7 years (10th-90th percentile 41.7-82.2 years); age at diagnosis was significantly higher with increasing severity of the categories of premalignant gastric lesions $(p<0.001)$. Within all categories of pre-malignant gastric lesions, female patients were significantly older than male patients $(\mathrm{p}<0.001)$.

\section{Incidence}

The number of patients with a first gastric biopsy increased by $3.7 \%$ per year over the study period, with a distinct maximum in 1998 (fig 1). For all patients who were biopsied, the proportion of patients with a first time diagnosis of a premalignant gastric lesion, however, gradually declined (fig 2). As expected, the proportion of patients with a diagnosis of a premalignant gastric lesion increased with age (fig 3).

The incidence of atrophic gastritis declined over the study period by $8.2 \%$ per year [ $95 \%$ CI $7.9 \%$ to $8.6 \%$ ], and the incidence of gastric dysplasia declined by $8.1 \%$ per year [ $95 \%$ CI $7.5 \%$ to $8.6 \%$ ]. These trends were similar for men and women ( $p$ values for differences were respectively 0.32 and 0.73 ). The decline in the number of diagnoses of intestinal metaplasia was $2.4 \%$ per year [ $95 \%$ CI $2.2 \%$ to $2.6 \%$ ] for women and $2.9 \%$ per year [95\% CI $2.7 \%$ to $3.1 \%$ ] for men ( $p$ value for difference 0.02 ). Age-period-cohort analysis was used to further investigate these drift values, that is, to differentiate between a period and a cohort effect. Significant period effects were demonstrated for atrophic gastritis ( $p$ values for men and women $<0.001)$ and intestinal metaplasia $(p=0.03$ for men, $p<0.001$ for women), but not for dysplasia ( $p=0.07$ for men, $p=0.93$ for women). The period pattern showed an accelerating decline after 1996 for both atrophic gastritis and intestinal metaplasia (fig 4). There were no apparent differences between men and women. In addition, the non-linear cohort effects for atrophic gastritis were significant for men $(p<0.001)$, but not for women $(p=0.22)$ (fig 5$)$. For men, the proportional decline increased over the whole period, except in the cohort born after 1965. However, the decline for women was almost linear. For intestinal metaplasia, the cohort effect for men was similar to the effect in men with atrophic gastritis, although less clear: the proportional annual change varied from $-2 \%$ to almost $-4 \%$ over the cohorts $(\mathrm{p}<0.001)$. In women, the decline became less in the younger cohorts and was almost 0 in cohorts born after 1950 ( $p<0.001$ for non-linear cohort effect). For dysplasia, men and women showed the same significant pattern again $(\mathrm{p}<0.001 ; \mathrm{p}=0.004)$ : a sharper decline for younger cohorts except the cohort born after 1965, possibly because the occurrence rates are low.

\section{DISCUSSION}

This study shows that pre-malignant gastric lesions are frequently encountered in routine biopsies obtained during upper GI endoscopies in a Western population, especially in older people. Atrophic gastritis is the most common premalignant gastric condition, as it can be assumed that all subsequent stages have underlying atrophic changes of the gastric mucosa. However, the incidence of these lesions has declined significantly over the past 15 years. The PAC increase in incidence in male patients was found to become stronger for men born after 1920. This matches the declining gastric cancer incidence in the Netherlands in the past 20 years and is thought 
Table 1 Baseline characteristics

\begin{tabular}{|c|c|c|c|c|}
\hline & Total & $\begin{array}{l}\text { Atrophic } \\
\text { gastritis }\end{array}$ & $\begin{array}{l}\text { Intestinal } \\
\text { metaplasia }\end{array}$ & Dysplasia \\
\hline $\begin{array}{l}\text { Number of patients } \\
\text { (n) (\%) }\end{array}$ & 97732 & $\begin{array}{l}23278 \\
(24 \%)\end{array}$ & $\begin{array}{l}65937 \\
(67 \%)\end{array}$ & $\begin{array}{l}8517 \\
(9 \%)\end{array}$ \\
\hline Male/female & $1.0 / 1.0$ & $1.0 / 1.2$ & $1.0 / 0.9$ & $1.0 / 0.8$ \\
\hline \multicolumn{5}{|l|}{ Age (years) } \\
\hline Median & 65.7 & 60.8 & 66.5 & 69.2 \\
\hline $\begin{array}{l}\text { 10th-90th } \\
\text { percentile }\end{array}$ & $41.7-82.2$ & $34.3-80.9$ & $44.3-82.4$ & $46.1-83.7$ \\
\hline
\end{tabular}

first of all to be related to a decrease in the prevalence of $H$ pylori infection in younger cohorts. ${ }^{16-18}$ In the Netherlands, the prevalence of $H$ pylori has declined from approximately $40 \%$ to $32 \%$ during the investigated period based on serological analyses of 918 and 1600 subjects in 1989 and 2006, respectively, in the age groups of 18 to 70 years. ${ }^{19} 20$

Similar observations have been made in a Finnish study, which showed a parallel decrease in the age-related prevalence of atrophic gastritis, intestinal metaplasia and gastric cancer in the period from 1977 to $1992 .{ }^{21}$ The decline in the incidence of all pre-malignant gastric lesions in our study is larger than the current decline of gastric cancer in the Netherlands, which was $2.13 \%$ [ $95 \%$ CI $1.83 \%$ to $2.43 \%$ ] annually over the period $1991-$ 2003. ${ }^{3}$ Therefore, a more rapid decline of gastric cancer incidence may be anticipated within the next decades. Based on our findings, a decline of approximately $24 \%$ may be expected within the coming 10 years, which corresponds to a decreasing incidence from 6.9 to $5.2 / 100$ 000/year (WSR): this incidence is lower than the current incidence in any Western country. ${ }^{1}$

A cohort phenomenon for the prevalence of $H$ pylori has previously been demonstrated by analysing individual longitudinal data, which showed that the prevalence of chronic $H$ pylori-induced gastritis is much lower in younger birth cohorts and that $H$ pylori infection is rarely acquired after childhood. ${ }^{92}$ The presence of a similar cohort phenomenon in the incidence of pre-malignant gastric lesions confirms the central role of $H$ pylori in gastric carcinogenesis and gastric cancer cascade described by Correa. ${ }^{7}$ Although gastric carcinogenesis is a complex and multifactorial process, $H$ pylori infection rates in childhood can presumably predict the occurrence rates of premalignant lesions later in life and possibly even gastric cancer in different birth cohorts.

A cohort effect was clearly demonstrated in this study for male patients. For female patients, a nearly linear decline was demonstrated for the diagnosis of atrophic gastritis, whereas for intestinal metaplasia a decline was almost absent in younger birth cohorts. These findings can probably not be explained by a difference in $H$ pylori prevalence differences between the sexes. Conversely, lifestyle factors, predominantly smoking, are the most probable explanation for these findings, as smoking seems an important factor in gastric carcinogenesis. $^{23-25}$ Gender differences in smoking habits in consecutive cohorts were previously observed in an observational Dutch study. This study demonstrated that the relative risk of lung cancer, as an indicator of smoking, is declining for men born after 1914. In contrast, the risk of lung cancer, and smoking has steadily increased in women. ${ }^{26}$

In addition, we observed that pre-malignant gastric lesions were diagnosed at a significantly older age in female patients (fig 3). These findings are in accordance with the male predominance in gastric cancer incidence and imply that female patients enter the carcinogenic cascade at an older age. Similar observations have previously been made in patients with Barrett's oesophagus. ${ }^{27}$ The most probable explanations for our findings are greater use of non-steroidal anti-inflammatory drugs and less smoking in absolute numbers in female patients, although an influence of other factors, such as sex hormones, can not be excluded. ${ }^{26}$

Atrophic gastritis and intestinal metaplasia have traditionally been evaluated as one entity, in which intestinal metaplasia was considered evidence of atrophic gastritis since specialised glands had been replaced by intestinal crypts. ${ }^{28-30}$ As the multistep cascade of gastric carcinogenesis has increasingly been recognised, intestinal metaplasia is now mostly evaluated as a separate entity in accordance with the updated Sydney system. In this study, we also assessed atrophic gastritis and intestinal metaplasia separately. This approach seems valid, as significant differences existed between both groups. For instance, patients with an initial diagnosis of intestinal metaplasia were significantly older than patients with atrophic gastritis $(p<0.001)$. Nevertheless, as only the most severe diagnosis at baseline is evaluated in this study, the incidence of atrophic gastritis has probably been underestimated. For a true calculation of the incidence of atrophic gastritis, the numbers of atrophic gastritis, intestinal metaplasia and dysplasia may be added, as it can be assumed that all patients with intestinal metaplasia suffer from atrophic gastritis and similarly, dysplasia in intestinal metaplasia-negative patients can be considered an exception. These calculations result in a decline of $4.4 \%$ per year [ $95 \%$ CI $4.2 \%$ to $4.6 \%$ ] for men and women with atrophic changes of the gastric mucosa, and a decline of $3.4 \%$ per year [ $95 \%$ CI $3.1 \%$ to $3.6 \%$ ] for men and $2.9 \%$ per year [ $95 \%$ CI $2.7 \%$ to $3.2 \%$ ] for women with intestinal metaplasia.

Potential weaknesses of our study are first that this study has been based on the number of histological diagnoses of premalignant lesions after upper GI endoscopy. Our findings could

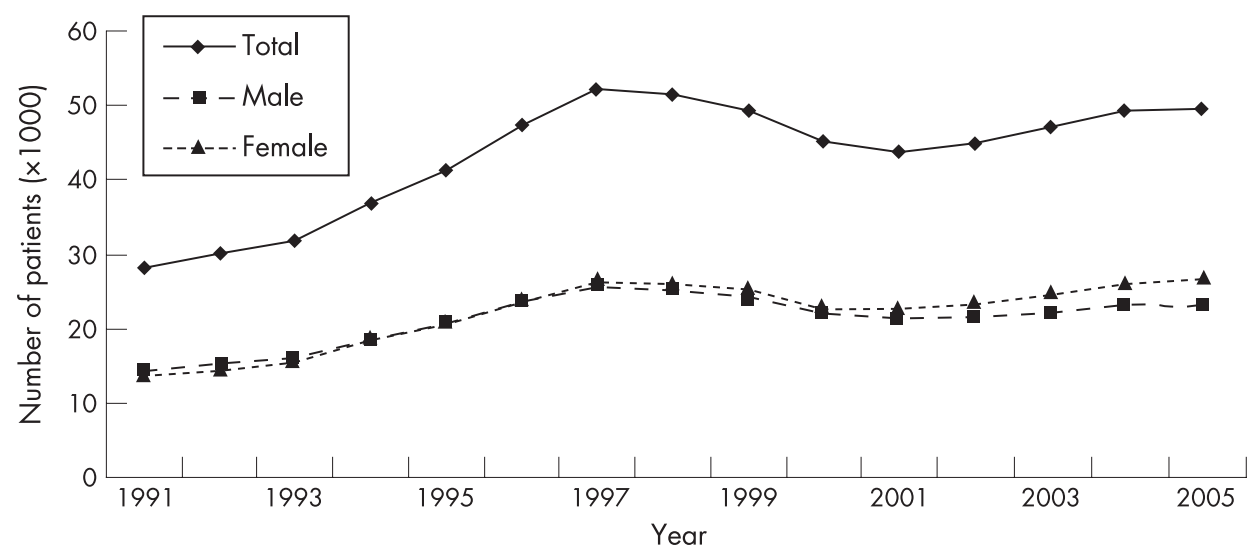

Figure 1 Number of patients with a first gastric biopsy during investigated period 1991-2005. 


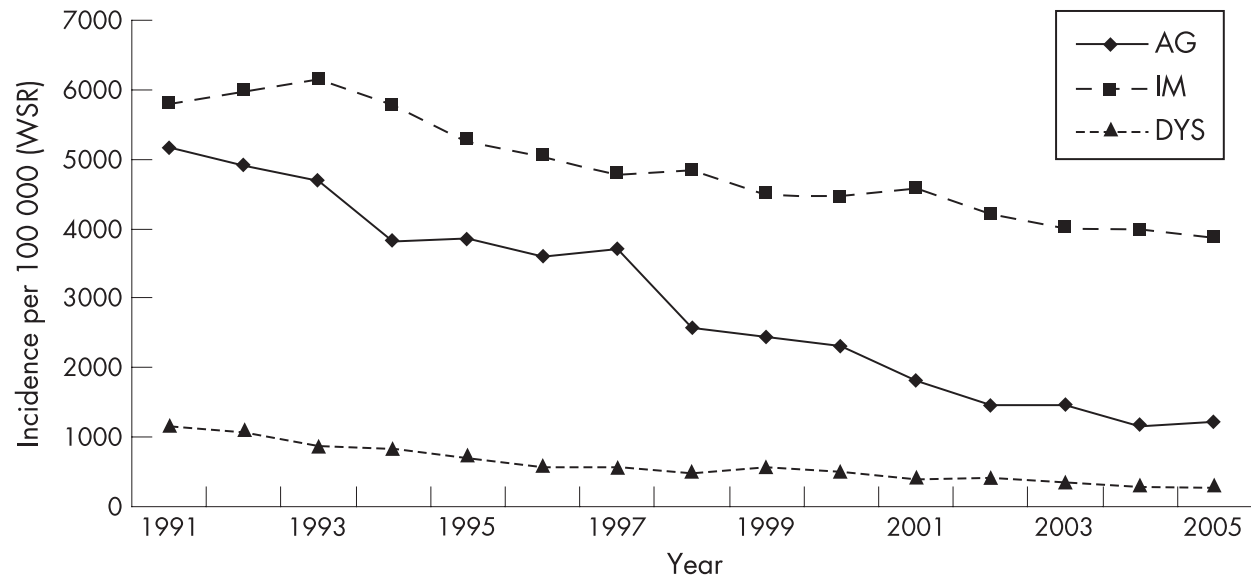

Figure 2 The prevalence of atrophic gastritis, intestinal metaplasia and dysplasia (WSR) relative to total number of patients with a first gastric biopsy over time. AG: atrophic gastritis; DYS: dysplasia; IM: intestinal metaplasia; WSR: world standardised rate. be influenced by altered indications for upper GI endoscopy over the past 15 years or by an altered attitude towards obtaining biopsies from macroscopic normal gastric mucosa, for example, increasing interest in diagnosing $H$ pylori for which biopsies from both antrum and corpus are at present commonly obtained. In case the total number of patients with a first gastric biopsy increases, a merely relative decline in the number of diagnoses of pre-malignant lesions could have been misinterpreted as a declining incidence of pre-malignant diagnoses in our study. However, the number of patients with a first gastric biopsy increased sharply before 1998, and thereafter a decline was followed by a slower incline until 2005 (fig 1). This trend has previously been observed, and is most likely explained by the introduction of Dutch general practitioner guidelines on dyspepsia in 1993, with a revision in 1996, in which restrictions in referrals for upper GI endoscopy were advised. ${ }^{31}$ This non-linear pattern was not observed in our period analysis of pre-malignant gastric lesions. Therefore, a true decline in the incidence of pre-malignant gastric lesions has been demonstrated in this study.
Second, changing trends in the assessment of gastric biopsies by pathologists could have attributed to the declining incidence of pre-neoplastic conditions of the stomach. The histological diagnosis and grading of pre-malignant gastric lesions have been subject of debate for years. At present, the updated Sydney system is most commonly used to grade gastritis. ${ }^{32}$ In this classification system several features of inflammation, atrophy and intestinal metaplasia are being assessed individually. Grading of gastric dysplasia is now in particular performed by means of the (updated) Vienna classification. ${ }^{33}{ }^{34}$ At present, dysplasia is commonly defined as intraepithelial or noninvasive neoplasia. We preferred to maintain the term dysplasia in this study, as this was the original diagnosis in our cases and was used as a search term, but it should be recognised that currently pathologists refer to these lesions as intraepithelial or non-invasive neoplasia. Although it has been shown that considerable differences between pathologists on diagnosing pre-malignant gastric lesions still exist, it can be speculated that the use of these specific grading classifications led to an increase in inter-observer agreement in more recent years. ${ }^{35-38}$

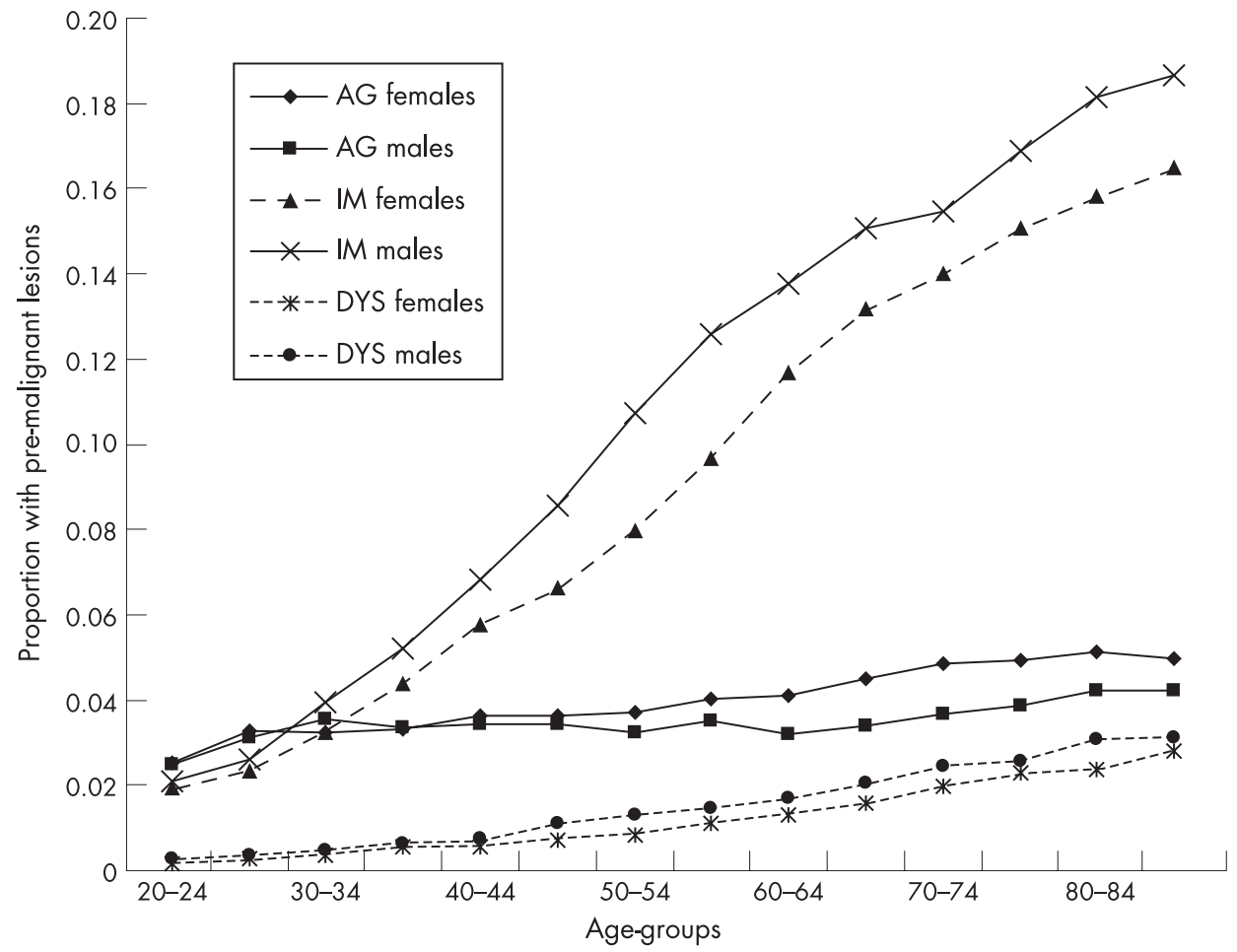

Figure 3 Proportion of patients with premalignant gastric lesions relative to total number of patients with a first gastric biopsy within 5-year age groups for total cohort and complete study period. AG: atrophic gastritis; DYS: dysplasia; IM: intestinal metaplasia. 

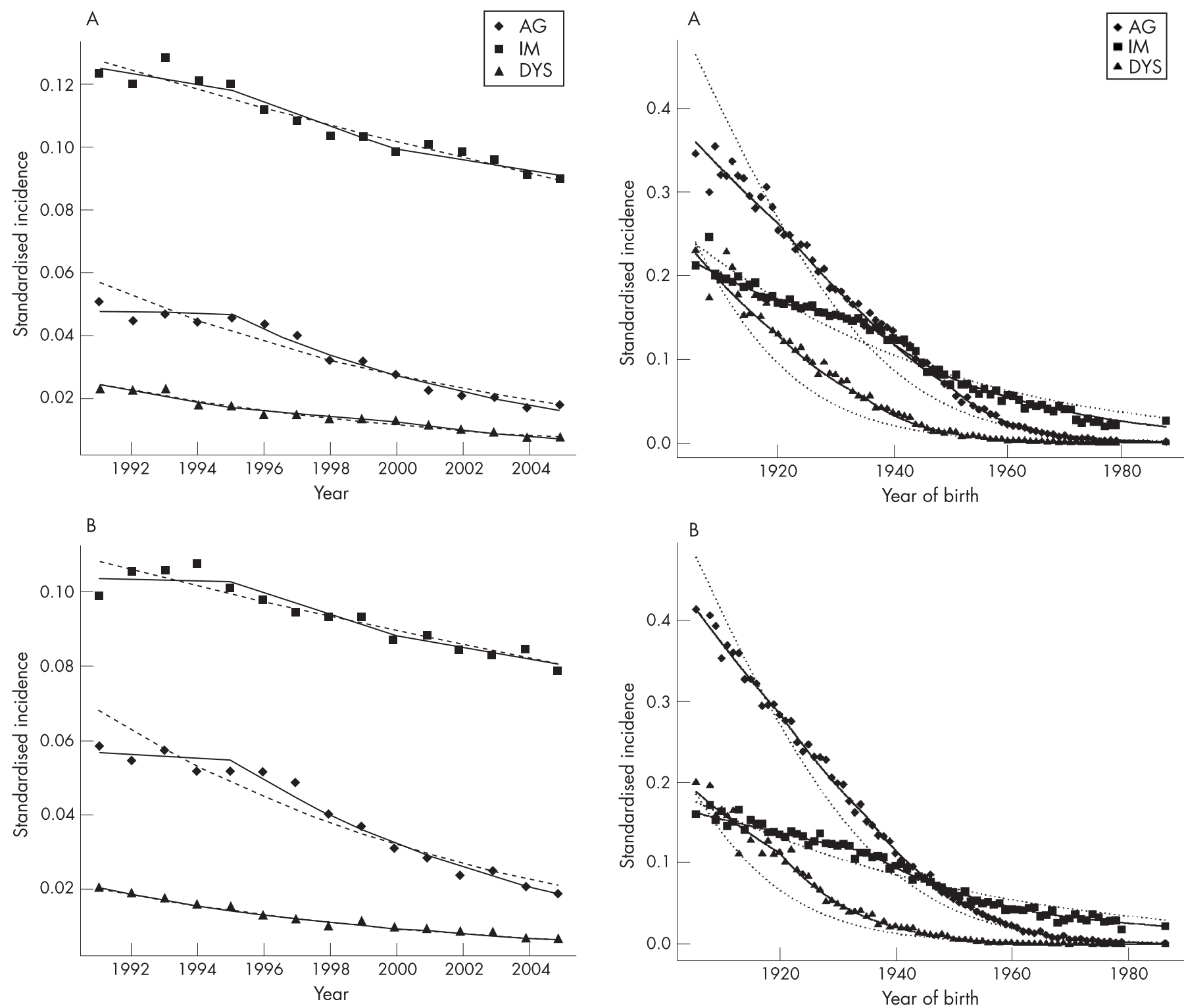

Figure 4 Period effects for pre-malignant gastric lesions for (A) male patients and (B) female patients. Symbols refer to age standardised prevalences, using the study population as standard. Lines are the linear splines from the age-period model; dotted lines show the results of the drift model, that is, the situation where there is a constant PAC over the whole range of years. AG: atrophic gastritis; DYS: dysplasia; IM: intestinal metaplasia.

Stricter criteria for assessment of dysplasia have been proposed recently, which in theory may have contributed to a decline in the number of new cases in recent years. ${ }^{34}{ }^{39}$ Yet, it is unclear whether this truly had any effect on the observed declining incidence of dysplasia, as a clear period effect has not been demonstrated in this study. Furthermore, the fact that over the complete period of observation less than $10 \%$ of all patients diagnosed with pre-malignant abnormalities of their gastric mucosa were classified as having dysplasia supports the restrictive use of this diagnosis over the whole time period.

On the other hand, the declining incidence of atrophic gastritis and intestinal metaplasia partly results from a period effect. This study showed an accelerating decline after 1996 for these diagnoses. This finding can in part be related to changing histological definitions, in particular with respect to atrophic gastritis in relation to the introduction of the updated Sydney system around 1996. ${ }^{32}$ However, as such an effect is less likely

Figure 5 Cohort effects for pre-malignant gastric lesions for (A) male patients and (B) female patients. Symbols refer to age standardised prevalences, using the study population as standard. Lines are the linear splines from the age-cohort model; dotted lines show the results of the drift model, that is, the situation where there is a constant PAC over the whole range of birth years. AG: atrophic gastritis; DYS: dysplasia; IM: intestinal metaplasia.

for intestinal metaplasia as a relatively straightforward diagnosis not affected by changing histological definitions, other effects must also have played a role. This can in part have been due to the widespread introduction of $H$ pylori diagnosis and treatment starting in the early 1990s.

Moreover, it is plausible that indications for upper GI endoscopy, for example, symptoms, led to a specific selection of the general population in this study. Therefore, these data are only suited to studying prevalence trends within patients with an indication for upper GI endoscopy. However, as differences in prevalence between our population and the general population are presumably small, it can be assumed that trend, period and cohort patterns observed here do reflect incidence patterns in general. To evaluate prevalence in the general Dutch population a prospective endoscopic populationbased study would be necessary. However, this study design would evidently be time-consuming and costly. Possibly, 
serological markers can serve as an alternative for this purpose when evaluating atrophic gastritis, however, its value in diagnosing intestinal metaplasia and dysplasia is still unclear. ${ }^{40}$

Unfortunately, we could not evaluate the number of biopsies and intragastric distribution of pre-malignant lesions in this study. For instance, it would be interesting to evaluate whether extension has decreased in subsequent periods and whether this extension progresses with age. At present, the Sydney system recommends two biopsies from the corpus, two from the antrum and one from the incisura angularis during gastroscopy to classify and grade gastritis. However, for the purpose of evaluation of pre-malignant lesions a more extensive biopsy scheme may be required. As knowledge of the exact intragastric distribution of pre-malignant lesions should guide biopsy sampling during surveillance endoscopy, future research should be focused on clarification of the extension and distribution of pre-malignant gastric lesions.

In conclusion, the incidence of pre-malignant gastric lesions is declining in countries with a low gastric cancer incidence within subsequent birth cohorts, probably caused by a declining incidence of $H$ pylori infections. Therefore, a considerable further decline in gastric cancer incidence of at least $24 \%$ in the coming decade may be anticipated in these countries and this will occur without any specific intervention.

\section{Authors' affiliations}

A C de Vries, B E Hansen, E J Kuipers, Department of Gastroenterology and Hepatology, Erasmus MC University Medical Center, Rotterdam G A Meijer, N C T van Grieken, Department of Pathology, VU University Medical Center, Amsterdam

C W N Looman, Department of Public Health, Erasmus MC University Medical Center, Rotterdam

M K Casparie, Prismant, Utrecht, The Netherlands

B E Hansen, Department of Epidemiology and Biostatistics, Erasmus MC University Medical Center, Rotterdam

E J Kuipers, Department of Internal Medicine, Erasmus MC University Medical Center, Rotterdam

Competing interests: None.

\section{REFERENCES}

1 Ferlay J, Bray F, Pisani P, et al. GLOBOCAN 2002: Cancer Incidence, Mortality and Prevalence Worldwide. IARC CancerBase No 5 version 2.0. Lyon: IARCPress, 2004

2 IKCnet. www.ikcnet.nl, 2003.

3 Bowles MJ, Benjamin IS. ABC of the upper gastrointestinal tract: cancer of the stomach and pancreas. BMJ 2001;323:1413-6.

4 Uemura N, Okamoto S, Yamamoto S, et al. Helicobacter pylori infection and the development of gastric cancer. N Engl J Med 2001;345:784-9

5 IARC Working Group on the Evaluation of Carcinogenic Risks to Humans. Schistosomes, liver flukes and Helicobacter pylori. 7-14 June 1994, Lyon. IARC Monogr Eval Carcinog Risks Hum 1994;61:1-241.

6 Helicobacter and Cancer Collaborative Group. Gastric cancer and Helicobacter pylori: a combined analysis of 12 case control studies nested within prospective cohorts. Gut 2001;49:347-53.

7 Correa P. Human gastric carcinogenesis: a multistep and multifactorial process. First American Cancer Society Award Lecture on Cancer Epidemiology and Prevention. Cancer Res 1992;52:6735-40.

8 Kuipers EJ, Uyterlinde AM, Pena AS, et al. Long-term sequelae of Helicobacter pylori gastritis. Lancet 1995;345:1525-8.

9 Sipponen P, Helske T, Jarvinen P, et al. Fall in the prevalence of chronic gastritis over 15 years: analysis of outpatient series in Finland from 1977, 1985, and 1992. Gut 1994;35:1167-71

10 Weck MN, Brenner $\mathrm{H}$. Prevalence of chronic atrophic gastritis in different parts of the world. Cancer Epidemiol Biomarkers Prev 2006;15:1083-94.

11 Casparie M, Tiebosch T, Burger G, et al. Pathology databanking and biobanking in The Netherlands, a central role for PALGA, the nationwide histopathology and cytopathology data network and archive. Cell Oncol 2007;29:19-24.

12 Cote RA, Robboy S. Progress in medical information management. Systematized nomenclature of medicine (SNOMED). JAMA 1980;243:756-62.

13 Ahmad OE, Boschi-Pinto C, Lopez AD, et al. Age standardization of rates: a new WHO standard. GPE Discussion Paper Series: No.31. Geneva: WHO, 2000.
14 Clayton D, Schifflers E. Models for temporal variation in cancer rates. I: Ageperiod and age-cohort models, Stat Med 1987;6:449-67.

15 Clayton D, Schifflers E. Models for temporal variation in cancer rates. II: Ageperiod-cohort models, Stat Med 1987;6:469-81

16 Loffeld RJ, van der Putten AB. Changes in prevalence of Helicobacter pylori infection in two groups of patients undergoing endoscopy and living in the same region in the Netherlands. Scand J Gastroenterol 2003;38:938-41.

17 Mourad-Baars PE, Verspaget HW, Mertens BJ, et al. Low prevalence of Helicobacter pylori infection in young children in the Netherlands. Eur J Gastroenterol Hepatol 2007;19:213-6.

18 Roosendaal R, Kuipers EJ, Buitenwerf J, et al. Helicobacter pylori and the birth cohort effect: evidence of a continuous decrease of infection rates in childhood. Am J Gastroenterol 1997;92:1480-2.

19 Loffeld RJ, Stobberingh E, van Spreeuwel JP, et al. The prevalence of antiHelicobacter (Campylobacter) pylori antibodies in patients and healthy blood donors. J Med Microbiol 1990;32:105-9.

20 van Vuuren AJ, de Man RA, van Driel HF, et al. Seroprevalence of Helicobacter pylori in two asymptomatic Dutch populations. Gastroenterology 2006;130(Suppl 2):T1895.

21 Sipponen P, Kimura K. Intestinal metaplasia, atrophic gastritis and stomach cancer: trends over time. Eur J Gastroenterol Hepatol 1994;6(Suppl 1):S79-83

22 Kuipers EJ, Pena AS, van KG, et al. Seroconversion for Helicobacter pylori. Lancet 1993;342:328-31.

23 Kneller RW, You WC, Chang YS, et al. Cigarette smoking and other risk factors for progression of precancerous stomach lesions. J Natl Cancer Inst 1992;84:1261-6.

24 Kato I, Vivas J, Plummer M, et al. Environmental factors in Helicobacter pylorirelated gastric precancerous lesions in Venezuela. Cancer Epidemiol Biomarkers Prev 2004;13:468-76.

25 Russo A, Maconi G, Spinelli P, et al. Effect of lifestyle, smoking, and diet on development of intestinal metaplasia in $H$. pylori-positive subjects. Am J Gastroenterol 2001;96:1402-8.

26 Barendregt JJ, Looman CW, Bronnum-Hansen $\mathrm{H}$. Comparison of cohort smoking intensities in Denmark and the Netherlands. Bull World Health Organ 2002;80:26-32.

27 van Blankenstein M, Looman CW, Johnston BJ, et al. Age and sex distribution of the prevalence of Barrett's esophagus found in a primary referral endoscopy center. Am J Gastroenterol 2005; 100:568-76.

28 Cheli R, Santi L, Ciancamerla G, et al. A clinical and statistical follow-up study of atrophic gastritis. Am J Dig Dis 1973;18:1061-5

29 Ectors N, Dixon MF. The prognostic value of sulphomucin positive intestinal metaplasia in the development of gastric cancer. Histopathology 1986;10:1271-7

30 Siurala M, Lehtola J, Ihamaki T. Atrophic gastritis and its sequelae. Results of 1923 years' follow-up examinations. Scand J Gastroenterol 1974;9:441-6.

31 van Soest EM, Dieleman JP, Siersema PD, Sturkenboom MC, Kuipers EJ. Increasing incidence of Barrett's oesophagus in the general population. Gut 2005;54:1062-6.

32 Dixon MF, Genta RM, Yardley JH, et al. Classification and grading of gastritis. The updated Sydney System. International Workshop on the Histopathology of Gastritis, Houston 1994. Am J Surg Pathol 1996;20:1161-81.

33 Dixon MF. Gastrointestinal epithelial neoplasia: Vienna revisited. Gut 2002;51:130-1

34 Schlemper RJ, Riddell RH, Kato $Y$, et al. The Vienna classification of gastrointestinal epithelial neoplasia. Gut 2000;47:251-5.

35 Chen $\mathbf{X Y}$, van Der Hulst RW, Bruno MJ, et al. Interobserver variation in the histopathological scoring of Helicobacter pylori related gastritis. J Clin Pathol 1999:52:612-5.

36 El-Zimaity HM, Graham DY, al-Assi MT, et al. Interobserver variation in the histopathological assessment of Helicobacter pylori gastritis. Hum Pathol 1996;27:35-41.

37 Offerhaus GJ, Price $A B$, Haot J, et al. Observer agreement on the grading of gastric atrophy. Histopathology 1999;34:320-5.

38 Rugge $\mathrm{M}$, Correa $\mathrm{P}$, Dixon MF, et al. Gastric mucosal atrophy: interobserver consistency using new criteria for classification and grading. Aliment Pharmacol Ther 2002;16:1249-59

39 Rugge M, Correa P, Dixon MF, et al. Gastric dysplasia: the Padova international classification. Am J Surg Pathol 2000;24:167-76.

40 Vaananen H, Vauhkonen M, Helske T, et al. Non-endoscopic diagnosis of atrophic gastritis with a blood test. Correlation between gastric histology and serum levels of gastrin-17 and pepsinogen I: a multicentre study, Eur J Gastroenterol Hepatol 2003;15:885-91.

\section{APPENDIX}

SNOMED-like codes used in the analysis:

- Atrophic gastritis: M58000, M58001, M58010.

- Intestinal metaplasia: M73000, M73200, M73320, M73321, M73300.

- Dysplasia: M74000, M74006, M74007, M74008, M74009. 